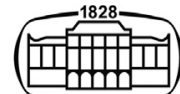

AKADÉMIAI KIADÓ

\title{
Effect of genetic and environmental influences on hepatic steatosis: A classical twin study based on computed tomography
}

IMAGING

\section{ORIGINAL ARTICLE}

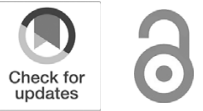

IMAGING 12 (2020) 1, 15-20

DOI: $10.1556 / 1647.2020 .00006$

(c) 2020 The Author(s)

*Corresponding author. BajcsyZsilinszky Hospital and Outpatient Department, 89-91 Maglodi ut, Budapest, H-1106, Hungary. Tel.: +36209282445.

E-mail: gyjermendy@gmail.com
GYÖRGY JERMENDY ${ }^{1 *}$, MÁRTON KOLOSSVÁRY ${ }^{2}$, IBOLYA DUDÁS ${ }^{2}$, ÁDÁM L. JERMENDY ${ }^{2}$, ALEXISZ PANAJOTU ${ }^{2}$, IMRE F. SUHAI ${ }^{3}$, ZSÓFIA D. DROBNI ${ }^{2}$, JÚLIA KARÁDY ${ }^{2,4}$, ÁDÁM D. TÁRNOKI ${ }^{3}$, DÁVID L. TÁRNOKI ${ }^{3}$, SZILARD VOROS ${ }^{5}$, BÉLA MERKELY ${ }^{2}$ and PÁL MAUROVICH-HORVAT ${ }^{2,3}$

\author{
${ }^{1}$ Bajcsy-Zsilinszky Hospital and Outpatient Department, Budapest, Hungary \\ ${ }^{2}$ MTA-SE Cardiovascular Imaging Research Group, Heart and Vascular Center, Semmelweis \\ University, Budapest, Hungary \\ ${ }^{3}$ Department of Radiology, Medical Imaging Centre, Semmelweis University, Budapest, \\ Hungary \\ ${ }^{4}$ Cardiovascular Imaging Research Center, Massachusetts General Hospital, Harvard Medical School, \\ Boston, MA, USA \\ ${ }^{5}$ Global Genomics Group, Atlanta, GA, USA
}

Received: April 27, 2020 • Accepted: May 16, 2020

\section{ABSTRACT}

Background and aims: Non-alcoholic fatty liver disease (NAFLD) increases cardiovascular morbidity and mortality, and carries poor long-term hepatic prognosis. Data about the role of genetic and environmental factors in the hepatic lipid accumulation are limited. The aim of the study was to evaluate the genetic and environmental impact on the hepatic lipid accumulation within a cohort of adult twin pairs.

Patients and methods: We investigated 182 twin subjects [monozygotic (MZ, $n=114)$ and dizygotic (DZ, $n=68$ ) same-gender twins (age $56.0 \pm 9.6$ years; BMI $27.5 \pm 5.0 \mathrm{~kg} / \mathrm{m}^{2}$; females $65.9 \%)$ ] who underwent computed tomography (CT) with a 256-slice scanner. Using nonenhanced CT-images, we calculated the average value of hepatic attenuation [expressed in Hounsfield unit (HU)] suggesting hepatic lipid content. Crude data were adjusted to age, sex, BMI and $\mathrm{HbAlc}$ values. Intra-pair correlations were established, and structural equation models were used for quantifying the contribution of additive genetic (A), common environmental (C) and unique environmental (E) components to the investigated phenotype.

Results: The study cohort represented a moderately overweight, middle-aged Caucasian population. There was no significant difference between $\mathrm{MZ}$ and $\mathrm{DZ}$ twin subjects regarding hepatic CT-attenuation $(57.9 \pm 12.6 \mathrm{HU}$ and $59.3 \pm 11.7 \mathrm{HU}$, respectively; $p=0.747)$. Age, sex, BMI and $\mathrm{HbAlc}$ adjusted co-twin correlations between the siblings showed that MZ twins have stronger correlations of $\mathrm{HU}$ values than $\mathrm{DZ}$ twins $(\mathrm{rMZ}=0.592, p<0.001 ; \mathrm{rDZ}=0.047, p=0.690$, respectively). Using the structural equation model, a moderate additive genetic dependence (A: $38 \%, 95 \%$ CI 15-58\%) and a greater unique environmental influence (E: 62\%, 95\% CI 42-85\%) was found. Common environmental influence was not identified (C: $0 \%)$.

Conclusion: The results of our classical CT-based twin study revealed moderate genetic and greater environmental influences on the phenotypic appearance of hepatic steatosis, commonly referred to as NAFLD. Favorable changes of modifiable environmental factors are of great importance in preventing or treating NAFLD. 


\section{Introduction}

Hepatic lipid accumulation in the absence of regular alcohol intake is commonly referred to as non-alcoholic fatty liver disease (NAFLD). In recent years, NAFLD has become increasingly more prevalent, affecting about $25 \%$ of adults worldwide, carrying severe vascular and hepatic outcomes [13]. It has been demonstrated previously that NAFLD might increase cardiovascular risk. In the Framingham Heart Study fatty liver was associated with several cardiovascular risk factors even after adjustment for other fat compartments [4]. Clinical studies have documented independent associations between NAFLD and increased incidence of cardiovascular events $[5,6]$. Furthermore, NAFLD may progress to NASH (nonalcoholic steatohepatitis) and ultimately to cirrhosis, hepatocellular carcinoma, and liver failure [7]. NAFLD is often associated with obesity, type 2 diabetes, hyperlipidemia, hypertension, insulin resistance and, therefore, some authors have suggested that NAFLD should be considered as a component of the metabolic syndrome $[8,9]$.

In the clinical setting, various imaging modalities including ultrasonography, computed tomography (CT) and magnetic resonance imaging (MRI) have been used to investigate and quantify hepatic steatosis [10-16]. In daily clinical practice, ultrasonography is routinely used but it has high intra- and interobserver variability. Nevertheless, improvements in methodology resulted in more reliable results $[17,18]$. Notably, European guidelines for the management of NAFLD recommend using ultrasonography as a first choice for imaging in adults who are at risk for NAFLD [19]. Recently, CT-imaging for the measurement of hepatic steatosis has become increasingly popular in different research and clinical projects $[20,21]$. Importantly, hepatic steatosis is quantifiable on non-contrast-enhanced CT-images and presents with decreased attenuation values of the parenchyma due to the inverse relationship between hepatic fat content and hepatic attenuation [10]. Although MRI proved to be more accurate measurement for evaluating hepatic lipid content, the availability of this method is limited, and its cost is relatively high. Therefore, MRI is limited and reserved mainly for research and clinical trials [22]. Undoubtedly, the exact diagnosis of NAFLD needs liver biopsy and should be based on histopathological investigations. Nevertheless, this invasive method is very rarely justified in practice [23]. Clinicians should base their diagnosis on laboratory findings, biomarkers and results of imaging procedures. Comparative studies with histopathological investigations validated the reliability and usefulness of ultrasonography, unenhanced CT or MRI [13, 21, 22].

Despite the growing knowledge about the pathogenesis of NAFLD, the entire process is still not completely understood. Data about the contribution of genetic and environmental influences on the hepatic steatosis are limited [24, 25]. In an earlier study, our working group found only negligible role for heritability of NAFLD within healthy, middle-aged twin pairs, however, lipid accumulation was visualized by using ultrasonography with its inherent limitations [26]. Currently, we intended to assess the effect of genetic and environmental influences on hepatic lipid accumulation within a cohort of adult twin pairs, undergoing CT-examination. Therefore, our objective was to evaluate the relative contribution of genetics versus environmental factors to hepatic steatosis, using CT-images available in participants of the BUDAPEST-GLOBAL clinical twin study.

\section{Patients and methods}

\section{Study population}

This study was a prospective, single-center, classical twin study involving monozygotic [MZ] and dizygotic [DZ] same-gender twins of self-reported Caucasian ethnicity. The investigation was conducted under the name of BUDAPEST-GLOBAL (Burden of atherosclerotic plaques study in twins - Genetic Loci and the Burden of Atherosclerotic Lesions) study; participants had been co-enrolled with the large, international, multicenter Genetic Loci and the Burden of Atherosclerotic Lesions (GLOBAL) clinical study (www.ClinicalTrials.gov: NCT01738828) [27]. Detailed study description and enrollment criteria were published previously [28]. The total study population consisted of 202 adult twin subjects (101 twin pairs) of whom 122 were MZ and 80 were same-gender DZ twin subjects. Participants were recruited from the Hungarian Twin Registry [29]. The study was approved by the National Scientific and Ethics Committee (institutional review board number: ETT TUKEB 58401/2012/EKU [828/PI/12], Amendment-1: 12292/2013/EKU [165/2013] and was carried out according to the principles stated in The Declaration of Helsinki. All subjects provided written informed consent.

The present analysis included 91 twin pairs (182 twin subjects; 120 women, 62 men); 10 twin pairs from the original cohort were excluded due to inadequate image quality. For the assessment of zygosity a self-reported questionnaire was used [30] and, based on this, $57 \mathrm{MZ}$ and $34 \mathrm{DZ}$ same-gender twin pairs were investigated. Main clinical and laboratory findings of twin pairs are summarized in Table 1.

\section{Computed tomography (CT) scanning protocol}

Each subject underwent a non-contrast enhanced CT-scan of the heart with a longer caudal coverage for visualizing the upper part of the abdomen (256-slice CT-scanner; Philips Brilliance iCT, Best, The Netherlands). Details of the study protocol were reported in our design paper [28]. Of note, the native CT-image acquisition resulted in a small $(<1 \mathrm{mSv})$ radiation dose.

Hepatic parenchymal attenuation was measured to quantify hepatic lipid content (Fig. 1). We selected three circular regions of interest (ROI) with an area of at least $300 \mathrm{~mm}^{2}$ on three cross-sectional images at different hepatic levels (one in the right hepatic lobe above the portal vein, one in the right hepatic lobe below the portal vein, and one in the left lobe). Special attention was taken to avoid hepatic larger vascular 
Table 1. Demographic characteristics, medical history and clinical-laboratory data of twin subjects

\begin{tabular}{|c|c|c|c|c|}
\hline Variables & $\begin{array}{l}\text { Twin subjects } \\
\text { (total, } n=182 \text { ) }\end{array}$ & $\begin{array}{c}\text { Monozygotic (MZ) } \\
\text { twin subjects }(n=114)\end{array}$ & $\begin{array}{l}\text { Dizygotic }(\mathrm{DZ}) \text { twin } \\
\text { subjects }(n=68)\end{array}$ & $\begin{array}{l}p \text { Value (MZ vs. } \\
\text { DZ twins) }\end{array}$ \\
\hline \multicolumn{5}{|c|}{ Demographic characteristics, medical history and clinical data } \\
\hline Female $n(\%)$ & $120(65.9 \%)$ & $70(61.4 \%)$ & $50(73.5 \%)$ & 0.095 \\
\hline Age (years) & $56.0 \pm 9.6$ & $54.6 \pm 9.7$ & $58.5 \pm 8.9$ & 0.006 \\
\hline BMI $\left(\mathrm{kg} / \mathrm{m}^{2}\right)$ & $27.5 \pm 5.0$ & $27.6 \pm 4.8$ & $27.4 \pm 5.3$ & 0.848 \\
\hline Waist circumference $(\mathrm{cm})$ & $96.1 \pm 13.2$ & $96.0 \pm 13.6$ & $96.6 \pm 12.8$ & 0.758 \\
\hline Hypertension $n(\%)$ & $75(41.2 \%)$ & $46(40.4 \%)$ & $29(42.6 \%)$ & 0.760 \\
\hline Diabetes mellitus $n(\%)$ & $16(8.8 \%)$ & $11(9.6 \%)$ & $5(7.4 \%)$ & 0.600 \\
\hline Current smoker $n(\%)$ & $31(17.0 \%)$ & $19(16.6 \%)$ & $12(17.6 \%)$ & 0.864 \\
\hline \multicolumn{5}{|l|}{ Laboratory parameters } \\
\hline Fasting glucose $(\mathrm{mmol} / \mathrm{L})$ & $5.31 \pm 1.27$ & $5.34 \pm 1.47$ & $5.26 \pm 0.85$ & 0.629 \\
\hline HbAlc (\%) & $5.5 \pm 0.9$ & $5.6 \pm 1.0$ & $5.2 \pm 0.8$ & 0.008 \\
\hline Total cholesterol $(\mathrm{mmol} / \mathrm{L})$ & $5.54 \pm 1.08$ & $5.65 \pm 1.13$ & $5.38 \pm 0.99$ & 0.095 \\
\hline LDL-cholesterol (mmol/L) & $3.47 \pm 0.99$ & $3.55 \pm 1.06$ & $3.35 \pm 0.85$ & 0.180 \\
\hline HDL-cholesterol (mmol/L) & $1.60 \pm 0.38$ & $1.59 \pm 0.40$ & $1.64 \pm 0.34$ & 0.357 \\
\hline Triglycerides $(\mathrm{mmol} / \mathrm{L})$ & $1.57 \pm 1.06$ & $1.67 \pm 1.23$ & $1.39 \pm 0.65$ & 0.043 \\
\hline Serum creatinine $(\mu \mathrm{mol} / \mathrm{L})$ & $75.9 \pm 8.8$ & $79.6 \pm 8.8$ & $70.7 \pm 8.8$ & 0.161 \\
\hline $\operatorname{ALT}(\mathrm{U} / \mathrm{l})$ & $20.2 \pm 11.6$ & $21.4 \pm 12.7$ & $18.1 \pm 9.0$ & 0.043 \\
\hline AST (U/l) & $22.2 \pm 10.8$ & $23.0 \pm 12.1$ & $20.7 \pm 7.9$ & 0.129 \\
\hline GGT (U/l) & $34.8 \pm 39.1$ & $37.4 \pm 28.9$ & $30.6 \pm 39.4$ & 0.260 \\
\hline Insulin $(\mu \mathrm{U} / \mathrm{mL})$ & $7.9 \pm 7.5$ & $8.4 \pm 8.2$ & $7.1 \pm 6.0$ & 0.250 \\
\hline C-peptide (ng/mL) & $2.3 \pm 1.4$ & $2.2 \pm 1.3$ & $2.3 \pm 1.5$ & 0.635 \\
\hline hsCRP (ng/mL) & $2.9 \pm 4.5$ & $2.7 \pm 2.9$ & $3.1 \pm 6.4$ & 0.658 \\
\hline
\end{tabular}

Continuous variables are presented as mean $\pm \mathrm{SD}$, while categorical as $n(\%)$. P values represent two-sided $p$ values for independent $t$-tests done between the monozygotic (MZ) and dizygotic (DZ) twin groups. BMI: body mass index, hsCRP: high sensitive C-reactive protein, HbAlc: hemoglobinAlc, HDL: high-density lipoprotein, LDL: low-density lipoprotein, ALT: alanine aminotransferase, AST: aspartate aminotransferase, GGT: $\gamma$-glutamyl transpeptidase

structures [21]. For further analysis we used the mean values of CT-attenuation measured in three ROIs of the liver. Hepatic attenuation was measured by two radiologists (ZD, ÁLJ both with 5 years of experience with CT) in consensus. CT-attenuation was expressed in Hounsfield unit (HU). Readers were blinded for the zygosity of subjects investigated.

\section{Anthropometric data, medical history and laboratory analysis}

We recorded basic anthropometric parameters (weight, height, waist circumference) in each subject. Brachial blood pressure was measured prior to the CT-exam. We used questionnaires for evaluating past medical history and current lifestyle (smoking, dietary habits, physical activity). Fasting peripheral blood draw was performed before the CTexamination and we used standard methods in certified laboratory for assessing laboratory parameters.

\section{Statistical analysis}

For evaluating genetic and environmental influences on NAFLD, first we assessed co-twin correlations of $\mathrm{HU}$ in $\mathrm{MZ}$ and DZ pairs separately, and then we evaluated the

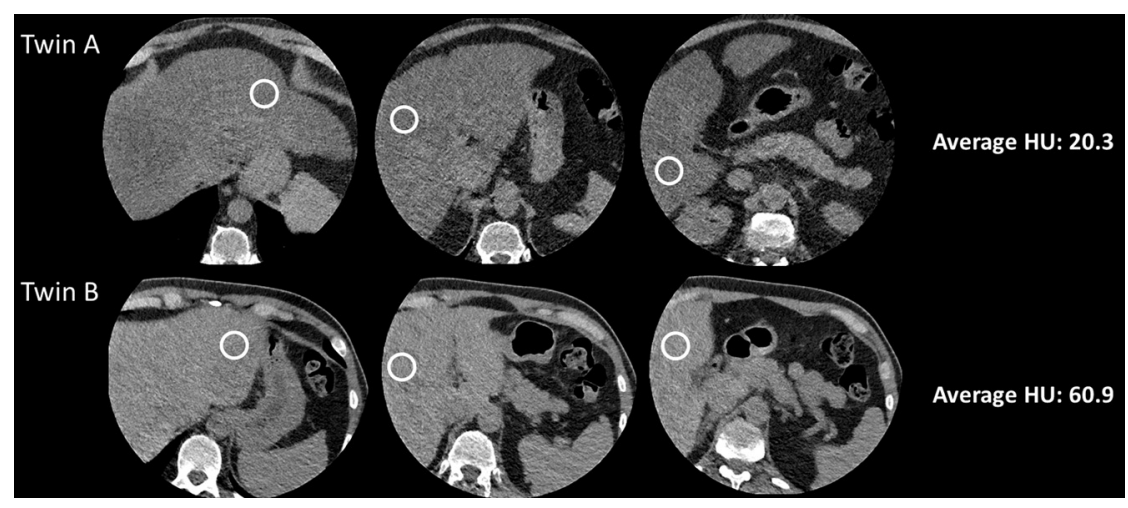

Figure 1. Evaluation of hepatic attenuation in a dizygotic twin pair. Measurements were performed in three regions of interest with at least $300 \mathrm{~mm}^{2}$ (white circles), and average values of the three measurements were used for analysis. HU: Hounsfield unit 
heritability using structural equation models often called ACE models. In the ACE model, the best fit model substantiates the quantification of genetic or environmental effects on phenotype investigated (in our study: hepatic lipid accumulation assessed by CT-attenuation, expressed in HU). All models were corrected for age, sex, BMI and HbAlc values. Log likelihood-based 95\% confidence intervals (CI) were calculated for all estimated parameters. The most parsimonious model best describing our data was found by eliminating, C (AE model), A (CE model) or AC (E model). Deterioration of fit was assessed using $-2 * \log$-likelihood values. All calculations were performed using $\mathrm{R}$ version 3.6.0. [31]. Twin modelling was performed using OpenMx version 2.12.2. [32]. Using the structural equation model, the effect of genetic and environmental influences on a given phenotype can be partitioned into additive genetic effects (A), common (or shared) environmental (C) and unshared (or unique) environmental (E) factors, which drive the variance in the phenotype for each twin. Additive genetic effect (A) is perfectly $(r=1.0)$ correlated across MZ twins and correlated less $(r=0.5)$ across DZ twins. Environmental components are grouped as common factors (C), which equally effect the siblings, and unique factors (E), which cause differences within families. Both $\mathrm{MZ}$ and $\mathrm{DZ}$ twins shared $100 \%$ of their $\mathrm{C}$ factors and none of their $\mathrm{E}$ factors. Since measurement error in the phenotype is also uncorrelated across measurements, it appears as part of the unique environmental component.

Continuous variables are expressed as mean \pm standard deviation (SD) whereas categorical variables are expressed as numbers and percentages. MZ and DZ twins were compared using Student's t-tests and Chi-square tests. Correlations were calculated using Pearson correlation coefficients. Descriptive statistics and correlations were calculated using IBM SPSS Statistics version 23 (IBM, Armonk, NY, USA). The level of significance was considered as $p<0.05$.

\section{Results}

Our study cohort (91 twin pairs, 182 twin subjects) represented a moderately overweight, middle-aged Caucasian population with a slight female predominance (Table 1). Mean values of laboratory data (including hepatic, renal and metabolic parameters) were within the normal range.

There was no significant difference between $\mathrm{MZ}$ and $\mathrm{DZ}$ twin subjects regarding hepatic CT-attenuation $(57.9 \pm 12.6$ $\mathrm{HU}$ and $59.3 \pm 11.7 \mathrm{HU}, p=0.747$, respectively).

Age-, sex-, BMI- and HbAlc-adjusted co-twin correlations between the siblings showed that MZ twins have stronger correlations of $\mathrm{HU}$ values than $\mathrm{DZ}$ twins $(\mathrm{rMZ}=$ $0.592, p<0.001 ; \mathrm{rDZ}=0.047, p=0.690$, respectively).

Using the structural equation model, the role of environmental influences was greater (E: $62 \%, 95 \%$ CI $42-85 \%$ ) compared to a moderate, additive genetic dependence (A: $38 \%$, 95\% CI 15-58\%). Common environmental influence was not identified (C: $0 \%)$. Detailed model information is given in Table 2.

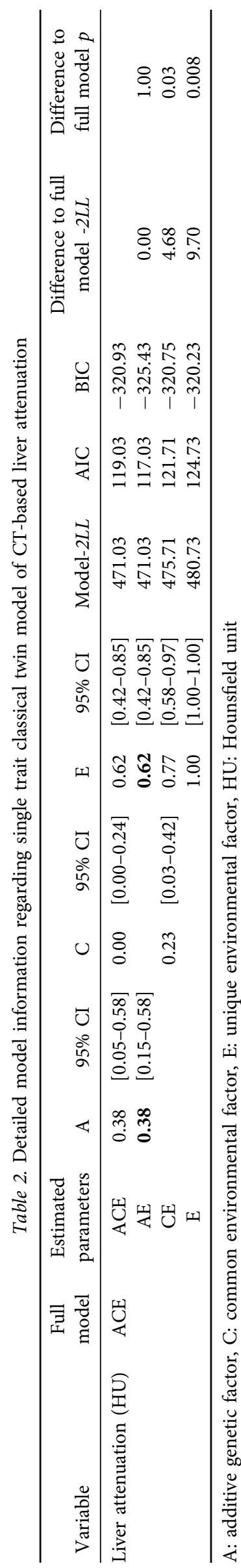




\section{Discussion}

Based on the results of our CT-based classical twin study, the contribution of unique environmental factors appears to have a greater role than genetic determinants in the development of hepatic steatosis.

We were uniquely positioned to address this question by performing a classical twin study which has value even in the current omics era of molecular genetic studies [33]. Our cohort consisted of middle-aged (56.0 \pm 9.6 years) adult twin subjects $(n=182)$, with a female predominance (65.9\%) and a higher number of MZ vs. DZ twin subjects ( $n$ $=114$ vs. $n=68$, respectively). These numbers are in line with former investigations as females and MZ twin pairs are more willing to participate in different research projects [34]. The sample size of our current investigation is also comparable to that of former classical twin studies [34].

We used non-enhanced CT-images for evaluating hepatic lipid accumulation, similarly to prior studies [10]. There is no consensus for CT-based criteria for NAFLD; both attenuation absolute values (expressed in HUs) and derived numbers (spleen-to-liver attenuation ratio or difference in attenuation values between liver and spleen) have been used [10, 21]. In our twin study, we preferred to use absolute numbers of HUs instead of derived ratios or differences. We considered more appropriate for assessing hepatic lipid content by measuring absolute attenuation values.

We found that environmental influences outweighed genetic influences in the development of hepatic lipid accumulation. Our current CT-based findings corroborate our previous investigation using ultrasonography [26], although the results of ACE models are numerically slightly different. Nevertheless, we expected a slight numerical difference as we used different methodology in a different twin population.

Our results have profound clinical implications. Since our study suggests that the development of NAFLD is predominantly influenced by environmental factors, the importance of lifestyle changes (including healthy diet, medical nutrition therapy, nutraceutical/dietary supplements, regular physical activity or behavioral intervention) is substantial in the primary prevention of NAFLD and related conditions $[35,36]$. In addition, bariatric surgery in obese people may reduce NAFLD [37]. Although several different drugs have shown some potential promising results in early clinical Phase- 2 and Phase- 3 trials, there are no currently approved drugs for the treatment and prevention of NAFLD and NASH $[38,39]$.

Our study has several limitations. Hepatic steatosis was estimated based on CT-attenuation, without any histological validation. Nevertheless, unenhanced CT-based evaluation of NAFLD is accepted, and histopathological correlations have been already published by others [21]. Furthermore, while biopsy-based steatosis measurements are severely hindered by "geographic miss" [40] our CT-based approach allows for the quantification of hepatic steatosis in a large, substantial portion of the liver. In our study, zygosity was classified using validated questionnaires; a method widely used in clinical twin studies [30]. Our study was performed in adult twin subjects of Caucasian population; therefore, the generalizability of our findings is limited.

In conclusion, our classical CT-based twin study documented moderate genetic and greater environmental influences on the phenotypic appearance of hepatic steatosis commonly referred to as NAFLD. Therefore, favorable changes of modifiable environmental factors are of great importance in preventing or treating NAFLD.

Funding source: The study was supported by a New Horizons Grant from the EASD (European Association from the Study of Diabetes) to György Jermendy, MD.

Authors' contribution: GJ, PMH conceived the study, GJ, ZDD drafted the manuscript, MK performed the statistical analysis, contributed to interpretation, ALJ, ZDD, AP, IFS, JK, ADT, DLT performed the clinical study, collected data, $\mathrm{PMH}$ provided continuous supervision of the study, SV, BM, $\mathrm{PMH}$ critically reviewed the manuscript with intellectual contribution to interpretation. GJ wrote the final version of manuscript. All authors take responsibility for the integrity of the data and the accuracy of the data analysis. All authors have read the final version of the manuscript and agreed to submit it to IMAGING for Open Access publication.

Conflict of interest: Szilard Voros, MD, is a shareholder in Global Genomics Group, LLC, and receives salary from Global Genomics Group, LLC. The authors have no other financial relationships, or conflicts of interest to disclose.

\section{REFERENCES}

[1] Younossi ZM, Koenig AB, Abdelatif D, Fazel Y, Henry L, Wymer M: Global epidemiology of nonalcoholic fatty liver disease. Metaanalytic assessment of prevalence, incidence, and outcomes. Hepatology 2016; 64: 73-84.

[2] Targher G, Day CP, Bonora E: Risk of cardiovascular disease in patients with nonalcoholic fatty liver disease. N Engl J Med 2010; 363: 1341-50.

[3] Bertot LC, Adams LA: Trends in hepatocellular carcinoma due to non-alcoholic fatty liver disease. Expert Rev Gastroenterol Hepatol 2019; 13: 179-87.

[4] Mellinger JL, Pencina KM, Massaro JM, Hoffmann U, Seshadri S, Fox CS, et al.: Hepatic steatosis and cardiovascular disease outcomes: an analysis of the Framingham Heart Study. J Hepatol 2015; 63: 470-6.

[5] Targher G, Bertolini L, Poli F, Rodella S, Scala L, Tessari R, et al.: Nonalcoholic fatty liver disease and risk of future cardiovascular events among type 2 diabetic patients. Diabetes 2005; 54: 3541-6.

[6] Hamaguchi M, Kojima T, Takeda N, Nagata C, Takeda J, Sarui H, et al.: Nonalcoholic fatty liver disease is a novel predictor of cardiovascular disease. World J Gastroenterol 2007; 13: 1579-84.

[7] Kumar R, Priyadarshi RN, Anand U: Non-alcoholic fatty liver disease: growing burden, adverse outcomes and associations. J Clin Transl Hepatol 2020; 8: 76-86. 
[8] Katsiki N, Perez-Martinez P, Anagnostis P, Mikhailidis DP, Karagiannis A: Is nonalcoholic fatty liver disease indeed the hepatic manifestation of metabolic syndrome? Curr Vasc Pharmacol 2018; 16: 219-27.

[9] Halmos T, Suba I: Non-alcoholic fatty liver disease, as a component of the metabolic syndrome, and its causal correlations with other extrahepatic diseases. Orv Hetil 2017; 158: 2051-61. [Hungarian].

[10] Schwenzer NF, Springer F, Schraml C, Stefan N, Machann J, Schick F: Non-invasive assessment and quantification of liver steatosis by ultrasound, computed tomography and magnetic resonance. J Hepatol 2009; 51: 433-45.

[11] Fabbrini E, Conte C, Magkos F: Methods for assessing intrahepatic fat content and steatosis. Curr Opin Clin Nutr Metab Care 2009; 12: $474-81$.

[12] Lee SS, Park SH: Radiologic evaluation of nonalcoholic fatty liver disease. World J Gastroenterol 2014; 20: 7392-402.

[13] Qayyum A, Chen DM, Breiman RS, Westphalen AC, Yeh BM, Jones KD: Evaluation of diffuse liver steatosis by ultrasound, computed tomography, and magnetic resonance imaging: which modality is best? Clin Imaging 2009; 33: 110-5.

[14] Stern C, Castera L: Non-invasive diagnosis of hepatic steatosis. Hepatol Int 2017; 11: 70-8.

[15] Castera L, Friedrich-Rust M, Loomba R: Noninvasive assessment of liver disease in patients with nonalcoholic fatty liver disease. Gastroenterology 2019; 156: 1264-81.

[16] Egresi A, Lengyel G, Hagymási K: Non-invasive assessment of fatty liver. Orv Hetil 2015; 156: 543-51. [Hungarian].

[17] Xia MF, Yan HM, He WY, Li XM, Li CL, Yao XZ, et al.: Standardized ultrasound hepatic/renal ratio and hepatic attenuation rate to quantify liver fat content: an improvement method. Obesity (Silver Spring, Md) 2012; 20: 444-52.

[18] Zhang B, Ding F, Chen T, Xia LH, Qian J, Lv GY: Ultrasound hepatic/renal ratio and hepatic attenuation rate for quantifying liver fat content. World J Gastroenterol 2014; 20: 17985-92.

[19] European Association for the Study of the Liver (EASL); European Association for the Study of Diabetes (EASD); European Association for the Study of Obesity (EASO): EASL-EASD-EASO Clinical Practice Guidelines for the management of non-alcoholic fatty liver disease. J Hepatol 2016; 64: 1388-402.

[20] Lee SW, Park SH, Kim KW, Choi EK, Shin YM, Kim PN, et al.: Unenhanced CT for assessment of macrovesicular hepatic steatosis in living liver donors: comparison of visual grading with liver attenuation index. Radiology 2007; 244: 479-85.

[21] Park SH, Kim PN, Kim KW, Lee SW, Yoon SE, Park SW, et al.: Macrovesicular hepatic steatosis in living liver donors: use of CT for quantitative and qualitative assessment. Radiology 2006; 239: 105-12.

[22] Fishbein M, Castro F, Cheruku S, Jain S, Webb B, Gleason T, et al.: Hepatic MRI for fat quantitation: its relationship to fat morphology, diagnosis, and ultrasound. J Clin Gastroenterol 2005; 39: 619-25.

[23] Danford CJ, Yao ZM, Jiang ZG: Non-alcoholic fatty liver disease: a narrative review of genetics. J Biomed Res 2018; 32: 389-400.

[24] Stefan N, Häring HU, Cusi K: Non-alcoholic fatty liver disease: causes, diagnosis, cardiometabolic consequences, and treatment strategies. Lancet Diabetes Endocrinol 2019; 7: 313-24.
[25] Zhou JH, Cai JJ, She ZG, Li HL: Noninvasive evaluation of nonalcoholic fatty liver disease: current evidence and practice. World J Gastroenterol 2019; 25: 1307-26.

[26] Tarnoki AD, Tarnoki DL, Bata P, Littvay L, Osztovits J, Jermendy G, et al.: Heritability of non-alcoholic fatty liver disease and association with abnormal vascular parameters: a twin study. Liver Int 2012; 32: 1287-93.

[27] Voros S, Maurovich-Horvat P, Marvasty IB, Bansal AT, Barnes MR, Vazquez G, et al.: Precision phenotyping, panomics, and system-level bioinformatics to delineate complex biologies of atherosclerosis: rationale and design of the 'Genetic Loci and the Burden of Atherosclerotic Lesions' study. J Cardiovasc Comput Tomogr 2014; 8: 442-51.

[28] Maurovich-Horvat P, Tárnoki DL, Tárnoki ÁD, Horváth T, Jermendy ÁL, Kolossváry M, et al.: Rationale, design, and methodological aspects of the BUDAPEST-GLOBAL Study (Burden of Atherosclerotic Plaques Study in Twins-Genetic Loci and the Burden of Atherosclerotic Lesions). Clin Cardiol 2015; 38: 699-707.

[29] Littvay L, Metneki J, Tarnoki AD, Tarnoki DL: The Hungarian twin registry. Twin Res Hum Genet 2013; 16: 185-9.

[30] Heath AC, Nyholt DR, Neuman R, Madden PA, Bucholz KK, Todd $\mathrm{RD}$, et al.: Zygosity diagnosis in the absence of genotypic data: an approach using latent class analysis. Twin Res 2003; 6: 22-6.

[31] R Core Team: R: A language and environment for statistical computing. R Foundation for Statistical Computing, Vienna, Austria, 2016. URL https://www.R-project.org/.

[32] Neale MC, Hunter MD, Pritikin JN, Zahery M, Brick TR, Kirkpatrick RM, et al.: OpenMx 2.0: extended structural equation and statistical modeling. Psychometrika 2016; 81: 535-49.

[33] van Dongen J, Slagboom PE, Draisma HH, Martin NG, Boomsma DI: The continuing value of twin studies in the omics era. Nat Rev Genet 2012; 13: 640-53.

[34] Elder SJ, Lichtenstein AH, Pittas AG, Roberts SB, Fuss PJ, Greenberg AS, et al.: Genetic and environmental influences on factors associated with cardiovascular disease and the metabolic syndrome. J Lipid Res 2009; 50: 1917-26.

[35] Ma J, Fox CS, Jacques PF, Speliotes EK, Hoffmann U, Smith CE, et al:: Sugar-sweetened beverage, diet soda, and fatty liver disease in the Framingham Heart Study cohorts. J Hepatol 2015; 63: 462-9.

[36] Katsagoni CN, Georgoulis M, Papatheodoridis GV, Panagiotakos DB, Kontogianni MD: Effects of lifestyle interventions on clinical characteristics of patients with non-alcoholic fatty liver disease: a meta-analysis. Metabolism 2017; 68: 119-32.

[37] Bower G, Toma T, Harling L, Jiao LR, Efthimiou E, Darzi A, et al.: Bariatric surgery and non-alcoholic fatty liver disease: a systematic review of liver biochemistry and histology. Obes Surg 2015; 25: 2280-9.

[38] Sumida Y, Yoneda M: Current and future pharmacological therapies for NAFLD/NASH. J Gastroenterol 2018; 53: 362-76.

[39] Firneisz G: Non-alcoholic fatty liver disease and type 2 diabetes mellitus: the liver disease of our age? World J Gastroenterol 2014; 20: 9072-89.

[40] Bedossa P, Patel K: Biopsy and noninvasive methods to assess progression of nonalcoholic fatty liver disease. Gastroenterol 2016; 150: 1811-22.e4.

Open Access statement. This is an open-access article distributed under the terms of the Creative Commons Attribution-NonCommercial 4.0 International License (https:// creativecommons.org/licenses/by-nc/4.0/), which permits unrestricted use, distribution, and reproduction in any medium for non-commercial purposes, provided the original author and source are credited, a link to the CC License is provided, and changes - if any - are indicated. 